\title{
The Influence of Personalised Sarcoma Care (PERSARC) Prediction Modelling on Clinical Decision Making in a Multidisciplinary Setting
}

\author{
H. S. Femke Hagenmaier ${ }^{1 D},{ }^{1}$ Annelies G. K. van Beeck, ${ }^{2,3}$ Rick L. Haas ${ }^{D},{ }^{4,5}$ \\ Veroniek M. van Praag, ${ }^{2}$ Leti van Bodegom-Vos, ${ }^{6}$ Jos A. van der Hage, ${ }^{7}$ Stijn Krol, ${ }^{4}$ \\ Frank M. Speetjens, ${ }^{8}$ Arjen H. G. Cleven, ${ }^{9}$ Ana Navas, ${ }^{10}$ Herman M. Kroon, ${ }^{10}$ \\ Rieneke G. Moeri-Schimmel, ${ }^{4}$ Nicolette A. C. Leyerzapf, ${ }^{2}$ and Michiel A. J. van de Sande $\mathbb{D D}^{2}$ \\ ${ }^{1}$ Department of Orthopaedic Surgery, Sint Maartenskliniek, Hengstdal 3, 6574 NA Ubbergen, Netherlands \\ ${ }^{2}$ Department of Orthopaedic Surgery, Leiden University Medical Centre, Albinusdreef 2, 2333 ZA Leiden, Netherlands \\ ${ }^{3}$ Department of Orthopaedic Surgery and Traumatology, University Hospital Antwerp, Wilrijkstraat 10, 2650 Edegem, Belgium \\ ${ }^{4}$ Department of Radiotherapy, Leiden University Medical Centre, Albinusdreef 2, 2333 ZA Leiden, Netherlands \\ ${ }^{5}$ Department of Radiotherapy, The Netherlands Cancer Institute, Plesmanlaan 121, 1066 CX Amsterdam, Netherlands \\ ${ }^{6}$ Department of Biomedical Data Sciences, Leiden University Medical Centre, Albinusdreef 2, 2333 ZA Leiden, Netherlands \\ ${ }^{7}$ Department of Surgery, Leiden University Medical Centre, Albinusdreef 2, 2333 ZA Leiden, Netherlands \\ ${ }^{8}$ Department of Clinical Oncology, Leiden University Medical Centre, Albinusdreef 2, 2333 ZA Leiden, Netherlands \\ ${ }^{9}$ Department of Pathology, Leiden University Medical Centre, Albinusdreef 2, 2333 ZA Leiden, Netherlands \\ ${ }^{10}$ Department of Radiology, Leiden University Medical Centre, Albinusdreef 2, 2333 ZA Leiden, Netherlands
}

Correspondence should be addressed to Michiel A. J. van de Sande; m.a.j.van_de_sande@lumc.nl

Received 21 September 2020; Revised 27 September 2021; Accepted 29 September 2021; Published 21 October 2021

Academic Editor: Cornelis Verhoef

Copyright (C) 2021 H. S. Femke Hagenmaier et al. This is an open access article distributed under the Creative Commons Attribution License, which permits unrestricted use, distribution, and reproduction in any medium, provided the original work is properly cited.

\begin{abstract}
Background. With soft-tissue sarcoma of the extremity (ESTS) representing a heterogenous group of tumors, management decisions are often made in multidisciplinary team (MDT) meetings. To optimize outcome, nomograms are more commonly used to guide individualized treatment decision making. Purpose. To evaluate the influence of Personalised Sarcoma Care (PERSARC) on treatment decisions for patients with high-grade ESTS and the ability of the MDT to accurately predict overall survival (OS) and local recurrence (LR) rates. Methods. Two consecutive meetings were organised. During the first meeting, 36 cases were presented to the MDT. OS and LR rates without the use of PERSARC were estimated by consensus and preferred treatment was recorded for each case. During the second meeting, OS/LR rates calculated with PERSARC were presented to the MDT. Differences between estimated OS/LR rates and PERSARC OS/LR rates were calculated. Variations in preferred treatment protocols were noted. Results. The MDT underestimated OS when compared to PERSARC in $48.4 \%$ of cases. LR rates were overestimated in $41.9 \%$ of cases. With the use of PERSARC, the proposed treatment changed for 24 cases. Conclusion. PERSARC aids the MDT to optimize individualized predicted OS and LR rates, hereby guiding patient-centered care and shared decision making.
\end{abstract}

\section{Introduction}

Over the last decades, prognostic modelling has found its way into prediction of survival and possible adverse events in sarcoma care. Among the first of those nomograms were the Memorial Sloan Kettering Cancer Center (MSKCC) Sarcoma Nomogram [1], SIN-system [2], and Sarculator [3]. The implementation of these prediction models was mostly 
restricted to individual patient and physician use, but has found its way into risk assessment and (possibly) risk-based management for this patient group. For example, high-risk patients identified by Sarculator were thought to benefit more from an intensified treatment setting including chemotherapy in high-grade soft-tissue sarcoma $[4,5]$.

In 2017, a prognostic model was developed to predict the cumulative incidence of overall survival (OS) and local recurrence (LR) rates for patients with primary high-grade Extremity Soft-Tissue Sarcoma (ESTS) [6]. This model, Personalised Sarcoma Care (PERSARC), was developed to support shared decision making between patients and physicians by providing better insight into individualised OS and LR estimates for different treatment options. PERSARC was subsequently made publicly available as a mobile application through app stores and the website of the Leiden University Medical Centre (LUMC), for both healthcare professionals and patients. Up to now, it has been used as an informative tool for general information only, as the implications of the use of PERSARC in clinical practice and its effect on shared decision making have yet to be established.

Due to the heterogeneity of ESTS [7], protocols for treatment decisions are not readily available or have not yet reached a broad consensus. To optimize patient outcome, management decisions are often formulated in multidisciplinary team (MDT) meetings which are still regarded the standard of care. Therefore, some treatment decisions are, to some extent, based on the conclusions of case discussions and collective experience of the MDT.

This study was conducted to evaluate the influence of PERSARC prediction on final treatment advice in everyday clinical practice, such as in an MDT. Furthermore, the ability of the specialised sarcoma healthcare professionals to accurately predict OS and LR rates for different treatment scenarios in patients with ESTS was determined.

\section{Materials and Methods}

2.1. Study Design. To establish the added value of PERSARC when implemented in clinical practice, two MDTs were organised. During each meeting, treatment options and their supposed consequential OS and LR rates were estimated by dedicated sarcoma healthcare professionals in consensus. For both MDTs, at a minimum, the same radiation oncologist, sarcoma surgeon, oncological orthopaedic surgeon, medical oncologist, radiologist, radiation oncologist, and a pathologist participated.

In the first MDT, a comprehensive case presentation was given, including information about age, gender, clinical symptoms, medical history, and results of physical examination. Furthermore, radiological imaging and the histological diagnosis were reviewed. Based on these data, a treatment proposal was formulated by the MDT, and the expected 5-year OS and LR with this treatment plan were estimated for each case without the use of PERSARC. Three months later, a second meeting was organised for the identical group of sarcoma specialists, presenting the same anonymised and randomized cases. The only additional information provided to the MDT was the optional treatment modalities with accompanying predictions of OS and LR rates calculated by PERSARC. Thereafter, once again, consensus was reached on a treatment advice, using the PERSARC estimates for OS and LR.

2.2. Study Population. All cases were selected from the Leiden University Medical Centre (LUMC) Sarcoma Registry. Patients eligible for review with PERSARC had a highgrade (Fédération Nationale des Centres de Lutte Contre le Cancer (FNCLCC) grade III) primary ESTS with a minimum follow-up of 1 year. Patients presenting with local recurrence and/or distant metastasis at initial diagnosis were excluded from this study. The results of magnetic resonance imaging (MRI) and additional imaging were discussed by the musculoskeletal (MSK) oncology radiologist and histology by a dedicated sarcoma pathologist.

2.3. PERSARC. Due to the heterogeneity of ESTS with more than 60 subtypes, PERSARC has grouped some subtypes together. The ESTS subtypes included were all high-grade (FNCLCC grades II and III): angiosarcoma, malignant peripheral nerve sheath tumor, synovial sarcoma, spindle cell sarcoma, myxofibrosarcoma, liposarcoma, leiomyosarcoma, malignant fibrous histiocytoma/undifferentiated pleomorphic sarcoma (pleomorphic), soft-tissue sarcomas not otherwise specified, and other. The updated PERSARC application (Version 2.0) was used being readily available through the website of the LUMC (https://www.lumc.nl/ org/oncologie-centrum/patienten/ziektes-en-

aandoeningen/wekedelentumor/persarc/). Patient characteristics needed for the PERSARC prediction model were age, sex, sarcoma size in centimetres, tumor depth, histological type, and histological grade of the tumor. PERSARC prediction modelling of OS and LR for different treatment modalities was updated and externally validated in 2021, using 3826 ESTS patients treated with curative intend. Patients were added to the model development cohort, and grade was included in the model. External validation was performed with data from 1111 patients treated at a single tertiary centre [7].

2.4. Outcomes. The following possible treatment scenarios were formulated, as presented in Table 1.

During the first MDT, OS and LR rates without the use of PERSARC were estimated by the MDT for the preferred treatment modality for each case. During the second MDT, OS and LR rates were calculated with PERSARC for all the aforementioned treatment regimens and presented to the MDT on screen. The difference between estimated OS/LR rates and PERSARC OS/LR rates was calculated ( $\triangle \mathrm{OS}$ and $\triangle \mathrm{LR}$ ), given as a positive or negative percentage to determine the degree of over- or underestimation of OS and LR rates by the MDT. All MDT estimations within 5\% of PERSARC predictions were considered normal variance. Furthermore, the chosen treatment proposals of the first and the second multidisciplinary sarcoma team meeting were compared for 
TABle 1: Treatment scenarios.

\begin{tabular}{lcc}
\hline Neoadjuvant radiotherapy & Surgery only & Adjuvant radiotherapy \\
\hline +Surgery R0 & $\mathrm{R} 0$ & + Surgery R0 \\
+Surgery R1 & $\mathrm{R} 1$ & + Surgery R1 \\
+Surgery R2 & $\mathrm{R} 2$ & +Surgery R2 \\
& Amputation & \\
\hline
\end{tabular}

each patient. Variations in preferred treatment regimens were noted.

2.5. Analysis. All statistical analyses were conducted with SPSS 20.0 (IBM SPSS Statistics for Windows, Version 20.0. Armonk, NY: IBM Corp). The Shapiro-Wilk test was used to test whether variables were normally distributed. In normally distributed data, mean and standard deviation were given. In nonnormally distributed data, median and range were used. A $p$ value of 0.05 was defined as statistically significant.

\section{Results}

3.1. Study Population. Thirty-six patients that met the study criteria were randomly chosen from the LUMC Sarcoma Registry and included in this study. There were 19 males and 17 females included for review, and mean age at diagnosis was 55.9 years. All patients presented with a high-grade ESTS without distant metastasis. The mean tumour size at presentation was $9.5 \mathrm{~cm}( \pm 5.9)$. Thirteen patients presented with a liposarcoma, 6 of which had a myxoid liposarcoma. All demographic data are presented in Table 2.

3.2. Overall Survival and Local Recurrence. Table 3 summarizes the treatments determined for each case and the accompanying estimated OS and LR rates without and with the use of PERSARC. The MDT proposed neoadjuvant radiotherapy followed by an intended R0 resection for 10 patients $(27.7 \%)$ and neoadjuvant radiotherapy followed by an expected R1 resection for 13 patients (36.1\%). In 2 cases (5.6\%), neoadjuvant radiotherapy followed by an R2 resection was expected based on patient and tumor characteristics. The MDT proposed surgery as monotherapy in 7 patients; in 2 cases (5.6\%), an expected R0 resection was performed, and in 5 patients (13.8\%), an amputation was advised. However, since amputation cannot yet be entered in the PERSARC app as a treatment option and, therefore, no reliable PERSARC prediction for OS/LR could be made, these data have been removed from this analysis.

Figure 1 shows the $\triangle \mathrm{OS}$ and $\triangle \mathrm{LR}$ for each individual case, expressed in percentages. A $5 \%$ positive or negative deviation from the absolute difference between PERSARC and the MDT was considered to be within normal range.

Regarding overall survival, the MDT underestimated OS when compared to PERSARC in $48.4 \%$ of cases $(15 / 31)$. They overestimated OS in $41.9 \%(13 / 31)$ and predicted OS correctly in $9.7 \%$ of cases $(3 / 31)$. For local recurrence, the MDT were more successful in their predictions, correctly estimating LR in $48.4 \%$ of our study population (15/31). It
Table 2: Patient demographics.

\begin{tabular}{lc}
\hline & $N(\%)$ \\
\hline Total & 36 \\
Age at diagnosis (mean (SD)) & $55.9(21.2)$ \\
\hline Gender (\%) & $19(53.0 \%)$ \\
$\quad$ Male & $17(47.0 \%)$ \\
$\quad$ Female & \\
\hline Depth (\%) & $13(36.0 \%)$ \\
Superficial & $23(64.0 \%)$ \\
Deep & $9.5( \pm 5.9)$ \\
\hline Size in cm (mean (SD)) & \\
\hline Histology (\%) & $4(11.1 \%)$ \\
Myxofibrosarcoma & $4(11.1 \%)$ \\
MPNST & $3(8.3 \%)$ \\
Synovial sarcoma & $2(5.6 \%)$ \\
MFH/UPS & $13(36.1 \%)$ \\
Liposarcoma & $10(27.8 \%)$ \\
$\quad$ Other &
\end{tabular}

${ }^{+}$Depth: relative to the investing fascia; N: number of patients; MFH/UPS: malignant fibrous histiocytoma/undifferentiated pleomorphic sarcoma; MPNST: malignant peripheral nerve sheath tumor.

overestimated LR in $41.9 \%(13 / 31)$ and underestimated LR in $9.7 \%$ of cases $(3 / 31)$.

3.3. PERSARC. Table 4 summarizes the proposed treatment regimens determined in the first meeting without the use of PERSARC and the second meeting using PERSARC. With the use of PERSARC, the proposed treatment changed in 24 cases $(66.7 \%)$. In 25 instead of 10 patients, the preferred treatment protocol was neoadjuvant RT and an intended R0 resection (69.4\% versus $27.7 \%$, respectively). Six additional patients would receive neoadjuvant RT and an expected R0 or R1 resection instead of surgery only.

In the surgery-only group, with the use of PERSARC, 7 additional patients were advised to receive an intended $\mathrm{R} 0$ instead of an expected R1 resection (16.7\% vs. $36.1 \%$, respectively). No intralesional resections were advised. Furthermore, there was a preference for amputation in only 1 patient $(2.8 \%)$, unlike $5(13.8 \%)$ without the use of PERSARC. With PERSARC, the MDT decided that there was no indication for adjuvant radiotherapy in patients that were regarded in need for radiotherapy in the first MDT without the use of PERSARC.

Table 5 further elaborates on the changes in the treatment protocol that were made with the use of PERSARC on a case-by-case basis.

\section{Discussion}

In this study designed to clinically evaluate the use of the PERSARC prediction model, we found that the use of PERSARC caused a variation in the preferred treatment option in $66.7 \%$ of cases. Furthermore, dedicated sarcoma specialists were better able to accurately predict local recurrence than overall survival (9.7\% versus $48.4 \%$, respectively) in a multidisciplinary setting. Overall survival was underestimated by the MDT in $48.4 \%$, compared to $9.7 \%$ 
TABLE 3: Estimated and calculated OS and LR for each case with and without PERSARC.

\begin{tabular}{|c|c|c|c|c|c|c|c|}
\hline \multirow[t]{2}{*}{ Case } & \multirow[t]{2}{*}{ Treatment proposal } & \multicolumn{2}{|c|}{$\begin{array}{c}\text { OS/LR MDT } \\
\text { estimation (\%) }\end{array}$} & \multicolumn{2}{|c|}{$\begin{array}{c}\text { OS/LR PERSARC } \\
\text { prognosis }(\%)\end{array}$} & \multicolumn{2}{|c|}{$\begin{array}{c}\Delta \text { prognosis }(\Delta-\% \\
\text { absolute })\end{array}$} \\
\hline & & OS & LR & OS & LR & $\Delta \mathrm{OS}$ & $\Delta \mathrm{LR}$ \\
\hline 1 & $\mathrm{R} 1$ & 92.5 & 30.0 & 74.2 & 22.8 & 18.3 & 7.2 \\
\hline 4 & Neoadjuvant RT, R1 & 82.5 & 5.0 & 41.1 & 15.8 & 41.4 & -10.8 \\
\hline 6 & Neoadjuvant RT, R1 & 27.5 & 20.0 & 14.0 & 20.4 & 13.5 & -0.4 \\
\hline 7 & Neoadjuvant RT, R1 & 70.0 & 12.5 & 55.4 & 13.6 & 14.6 & -1.1 \\
\hline 8 & $\mathrm{R} 0$ & 20.0 & 17.5 & 25.9 & 13.1 & -5.9 & 4.4 \\
\hline 9 & Neoadjuvant RT, R1 & 40.0 & 15.0 & 77.0 & 10.0 & -37.0 & 5.0 \\
\hline 10 & Neoadjuvant RT, R2 & 65.0 & 17.5 & 71.7 & 15.3 & -6.7 & 2.2 \\
\hline 13 & Neoadjuvant RT, R1 & 82.5 & 5.0 & 72.7 & 10.9 & 9.8 & -5.9 \\
\hline 14 & Neoadjuvant RT, R2 & 57.5 & 22.5 & 29.9 & 24.3 & 27.6 & -1.8 \\
\hline 15 & Neoadjuvant RT, R0 & 65.0 & 17.5 & 81.3 & 2.9 & -16.3 & 14.6 \\
\hline 16 & Neoadjuvant RT, R0 & 80.0 & 5.0 & 71.1 & 5.0 & 8.9 & 0.0 \\
\hline 17 & Neoadjuvant RT, R1 & 82.5 & 12.5 & 80.4 & 8.7 & 2.1 & 3.8 \\
\hline 18 & Neoadjuvant RT, R0 & 72.5 & 7.5 & 76.7 & 4.4 & -4.2 & 3.1 \\
\hline 19 & R0 & 70.0 & 45.0 & 54.7 & 13.9 & 15.3 & 31.1 \\
\hline 20 & Neoadjuvant RT, R0 & 72.5 & 10.0 & 79.0 & 2.3 & -6.5 & 7.7 \\
\hline 21 & Neoadjuvant RT, R0 & 80.0 & 5.0 & 77.0 & 4.8 & 3.0 & 0.2 \\
\hline 22 & Neoadjuvant RT, R0 & 75.0 & 10.0 & 57.6 & 6.6 & 17.4 & 3.4 \\
\hline 23 & Neoadjuvant RT, R1 & 62.5 & 12.5 & 49.2 & 10.0 & 13.3 & 2.5 \\
\hline 24 & $\mathrm{R} 1$, adjuvant RT & 17.5 & 55.0 & 37.1 & 29.9 & -19.6 & 25.1 \\
\hline 25 & Neoadjuvant RT, R1 & 40.0 & 22.5 & 70.7 & 7.8 & -30.7 & 14.7 \\
\hline 26 & Neoadjuvant RT, R1 & 60.0 & 20.0 & 79.7 & 9.3 & -19.7 & 10.7 \\
\hline 27 & Neoadjuvant RT, R1 & 60.0 & 10.0 & 71.8 & 10.0 & -11.8 & 0.0 \\
\hline 28 & $\mathrm{R} 2$ & 12.5 & 100.0 & 28.9 & 27.3 & -16.4 & 72.7 \\
\hline 29 & Neoadjuvant RT, R0 & 65.0 & 10.0 & 41.8 & 4.9 & 23.2 & 5.1 \\
\hline 30 & Neoadjuvant RT, R1 & 15.0 & 30.0 & 44.0 & 10.2 & -29.0 & 19.8 \\
\hline 31 & Neoadjuvant RT, R0 & 85.0 & 10.0 & 77.9 & 3.0 & 7.1 & 7.0 \\
\hline 32 & $\mathrm{R} 2$ & 5.0 & 30.0 & 17.1 & 52.8 & -12.1 & -22.8 \\
\hline 33 & Neoadjuvant RT, R1 & 20.0 & 20.0 & 53.1 & 12.9 & -33.1 & 7.1 \\
\hline 34 & Neoadjuvant RT, R1 & 35.0 & 25.0 & 59.4 & 9.4 & -24.4 & 15.6 \\
\hline 35 & Neoadjuvant RT, R0 & 60.0 & 10.0 & 66.6 & 5.5 & -6.6 & 4.5 \\
\hline 36 & Neoadjuvant RT, R0 & 65.0 & 10.0 & 79.9 & 4.3 & -14.9 & 5.7 \\
\hline
\end{tabular}

$\mathrm{RT}$ : radiotherapy.

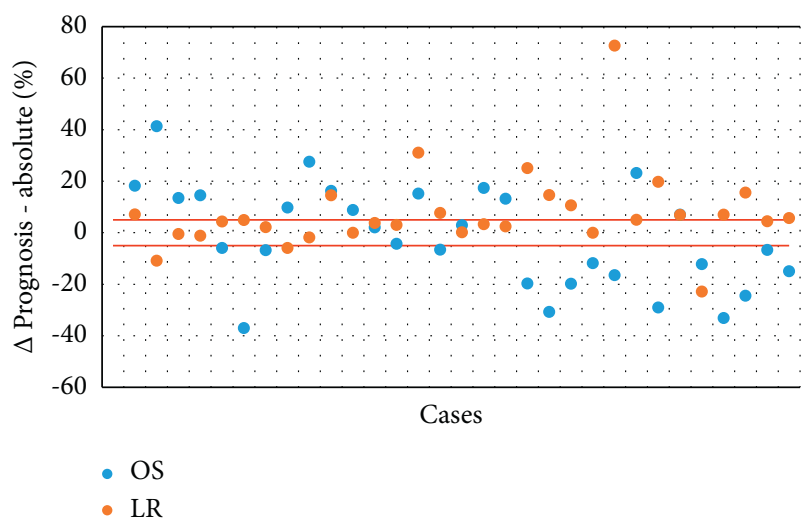

Figure 1: Differences in the prognosis of OS/LR.

underestimation for local recurrence. Although they predicted local recurrence accurately more often, the MDT still overestimated LR in the remainder (41.9\%) of our study population.

Due to its heterogeneity and complex nature with a diverse clinical behavior depending on subtype, several
TABLE 4: Chosen treatment options with and without the use of PERSARC.

\begin{tabular}{|c|c|c|}
\hline & $\begin{array}{c}\text { Without PERSARC, } N \\
(\%)\end{array}$ & $\begin{array}{c}\text { With PERSARC, } N \\
(\%)\end{array}$ \\
\hline \multicolumn{3}{|c|}{ Neoadjuvant radiotherapy } \\
\hline Surgery R0 & $10(27.7 \%)$ & $25(69.4 \%)$ \\
\hline Surgery R1 & $13(36.1 \%)$ & $6(16.7 \%)$ \\
\hline Surgery R2 & $2(5.6 \%)$ & $0(0.0 \%)$ \\
\hline \multicolumn{3}{|l|}{ Surgery } \\
\hline $1(2.8 \%)$ & Amputation & $5(13.8 \%)$ \\
\hline R0 & $2(5.6 \%)$ & $1(2.8 \%)$ \\
\hline $\mathrm{R} 1$ & $1(2.8 \%)$ & $3(8.3 \%)$ \\
\hline $\mathrm{R} 2$ & $2(5.6 \%)$ & $0(0.0 \%)$ \\
\hline \multicolumn{3}{|c|}{ Adjuvant radiotherapy } \\
\hline Surgery R0 & $0(0.0 \%)$ & $0(0.0 \%)$ \\
\hline Surgery R1 & $1(2.8 \%)$ & $0(0.0 \%)$ \\
\hline Surgery R2 & $0(0.0 \%)$ & $0(0.0 \%)$ \\
\hline
\end{tabular}

previous studies have emphasized the importance of multidisciplinary care and dedicated team meetings to optimize outcome in the treatment of ESTS [8-11]. Although some 
TABLE 5: Changes in the treatment protocol per case $(N=24)$ with the use of PERSARC.

\begin{tabular}{lcc}
\hline Case & Without PERSARC & With PERSARC \\
\hline 3 & Amputation & Neoadjuvant RT, R1 \\
4 & Neoadjuvant RT, R1 & Neoadjuvant RT, R0 \\
5 & Amputation & Neoadjuvant RT, R0 \\
6 & Neoadjuvant RT, R1 & Neoadjuvant RT, R0 \\
7 & Neoadjuvant RT, R1 & Neoadjuvant RT, R0 \\
8 & R0 & Neoadjuvant RT, R0 \\
9 & Neoadjuvant RT, R1 & Neoadjuvant RT, R0 \\
10 & Neoadjuvant RT, R2 & Neoadjuvant RT, R1 \\
11 & Amputation & Neoadjuvant RT, R0 \\
12 & Amputation & Neoadjuvant RT, R1 \\
13 & Neoadjuvant RT, R1 & Neoadjuvant RT, R0 \\
14 & Neoadjuvant RT, R2 & Neoadjuvant RT, R1 \\
19 & R0 & R1 \\
23 & Neoadjuvant RT, R1 & Neoadjuvant RT, R0 \\
24 & R1, adjuvant RT & R0 \\
25 & Neoadjuvant RT, R1 & Neoadjuvant RT, R0 \\
26 & Neoadjuvant RT, R1 & Neoadjuvant RT, R0 \\
27 & Neoadjuvant RT, R1 & Neoadjuvant RT, R0 \\
28 & Neoadjuvant RT, R1 & Neoadjuvant RT, R0 \\
30 & Neoadjuvant RT, R1 & R1 \\
31 & Neoadjuvant RT, R0 & Neoadjuvant RT, R1 \\
32 & R2 & Neoadjuvant RT, R0 \\
33 & Neoadjuvant RT, R1 & Neoadjuvant RT, R0 \\
34 & Neoadjuvant RT, R1 & Neoadjuvant RT, R0 \\
\hline
\end{tabular}

$\mathrm{RT}$ : radiotherapy.

have tried to address the quality and influence of MDT's on clinical decision making, none have assessed the predictive abilities of the multidisciplinary team and its members regarding the estimation of LR and OS rates in ESTS patients. In the current study, we found a slight overestimation of the local recurrence rate by the MDT (in 41.9\%), which may have led to a collective underestimation of overall survival. As a result, the MDT may have chosen a more individual patient-care-based approach in some patients, instead of a more aggressive approach with curative intent. The use of the PERSARC prediction model caused a change in preferred treatment modality in 24 patients (66.7\% of cases), mainly based on predicted higher overall survival rates by PERSARC. Therefore, PERSARC helps clinicians to estimate a more realistic prognosis of expected recurrence rates and life expectancy. This can potentially lead to a more frequent choice for limb salvage treatment, while maintaining comparable survival rates for these individual patients.

Looking at the different suggested treatment options, surgery with neoadjuvant radiotherapy was the treatment regimen most frequently preferred. Postoperative radiotherapy was completely discarded by the MDT as a potential treatment option (1 patient treated with adjuvant radiotherapy without PERSARC and none with PERSARC). Although the literature reports a poorer long-term functional outcome of the affected extremity for patients treated with postoperative radiotherapy, mainly due to the higher postoperative radiation dose, wound complications are much more common after preoperative radiotherapy [12-15]. As these short-term complications usually are manageable, they may be recognized as more acceptable, with better understanding of the predicted survival and local recurrence rates.

Even though PERSARC provides a validated prediction of LR and OS rates, there are some limitations that have to be considered [6]. First, the study population consisted of a relatively small number of patients, selected from the LUMC Sarcoma Registry, based on retrospective statistics. Second, the influence of comorbidity on overall survival is a wellknown prognostic factor [16], which has not yet been implemented in the PERSARC model. Although the dedicated sarcoma team was provided with data about clinical condition and known comorbidities, the influence on treatment effectiveness, overall survival, and expected quality of life still needs to be considered in the final (shared) decision making.

We conclude that this study illustrates the additional individualized value of the PERSARC prediction model in clinical decision making. PERSARC provides specialised medical sarcoma professionals with improved insight in predicted local recurrence rates and overall survival chances for ESTS patients regarding different treatment modalities. In this respect, it may prove to be a valuable tool toward patient-centered care and shared decision making.

\section{Data Availability}

There are no supporting data available for the current manuscript.

\section{Conflicts of Interest}

The authors declare no conflicts of interest.

\section{References}

[1] M. W. Kattan, D. H. Y. Leung, and M. F. Brennan, "Postoperative nomogram for 12-year sarcoma-specific death," Journal of Clinical Oncology, vol. 20, no. 3, pp. 791-796, 2002.

[2] P. Gustafson, M. Åkerman, T. A. Alvegård et al., "Prognostic information in soft tissue sarcoma using tumour size, vascular invasion and microscopic tumour necrosis-the SIN-system," European Journal of Cancer, vol. 39, no. 11, pp. 1568-1576, 2003.

[3] D. Callegaro, R. Miceli, S. Bonvalot et al., "Development and external validation of two nomograms to predict overall survival and occurrence of distant metastases in adults after surgical resection of localised soft-tissue sarcomas of the extremities: a retrospective analysis," The Lancet Oncology, vol. 17, no. 5, pp. 671-680, 2016.

[4] S. Pasquali, C. Colombo, S. Pizzamiglio et al., "High-risk soft tissue sarcomas treated with perioperative chemotherapy: improving prognostic classification in a randomised clinical trial," European Journal of Cancer, vol. 93, pp. 28-36, 2018.

[5] S. Pasquali, S. Pizzamiglio, N. Touati et al., "The impact of chemotherapy on survival of patients with extremity and trunk wall soft tissue sarcoma: revisiting the results of the EORTC-STBSG 62931 randomised trial," European Journal of Cancer, vol. 109, pp. 51-60, 2019.

[6] V. M. van Praag, A. J. Rueten-Budde, L. M. Jeys et al., “A prediction model for treatment decisions in high-grade extremity soft-tissue sarcomas: personalised sarcoma care 
(PERSARC)," European Journal of Cancer, vol. 83, pp. 313323, 2017.

[7] A. J. Rueten-Budde, "External validation and adaptation of a dynamic prediction model for patients with high-grade extremity soft tissue sarcoma," Journal of Surgical Oncology, vol. 123, no. 4, pp. 1050-1056, 2021.

[8] L. M. Nystrom, N. B. Reimer, J. D. Reith et al., "Multidisciplinary management of soft tissue sarcoma," Scientific World Journal, vol. 2013, Article ID 852462, 11 pages, 2013.

[9] B. Pillay, A. C. Wootten, H. Crowe et al., "The impact of multidisciplinary team meetings on patient assessment, management and outcomes in oncology settings: a systematic review of the literature," Cancer Treatment Reviews, vol. 42, pp. 56-72, 2016.

[10] G. W. Siegel, J. S. Biermann, R. Chugh et al., "The multidisciplinary management of bone and soft tissue sarcoma: an essential organizational framework," Journal of Multidisciplinary Healthcare, vol. 8, pp. 109-115, 2015.

[11] C. Taylor, J. Finnegan-John, and J. Green, "“No decision about me without me" in the context of cancer multidisciplinary team meetings: a qualitative interview study," BMC Health Services Research, vol. 14, no. 1, p. 488, 2014.

[12] E. Al-Absi, F. Farrokhyar, R. Sharma et al., "A systematic review and meta-analysis of oncologic outcomes of pre- versus postoperative radiation in localized resectable soft-tissue sarcoma," Annals of Surgical Oncology, vol. 17, no. 5, pp. 1367-1374, 2010.

[13] M. H. Abouarab, I. L. Salem, M. M. Degheidy et al., "Therapeutic options and postoperative wound complications after extremity soft tissue sarcoma resection and postoperative external beam radiotherapy," International Wound Journal, vol. 15, no. 1, pp. 148-158, 2018.

[14] E. H. Baldini, M. R. Lapidus, Q. Wang et al., "Predictors for major wound complications following preoperative radiotherapy and surgery for soft-tissue sarcoma of the extremities and trunk: importance of tumor proximity to skin surface," Annals of Surgical Oncology, vol. 20, no. 5, pp. 1494-1499, 2013.

[15] M. G. Stevenson, J. F. Ubbels, J. Slump et al., "Identification of predictors for wound complications following preoperative or postoperative radiotherapy in extremity soft tissue sarcoma," European Journal of Surgical Oncology, vol. 44, no. 6, pp. 816-822, 2018.

[16] J. Stairmand, L. Signal, D. Sarfati et al., "Consideration of comorbidity in treatment decision making in multidisciplinary cancer team meetings: a systematic review," Annals of Oncology, vol. 26, no. 7, pp. 1325-1332, 2015. 